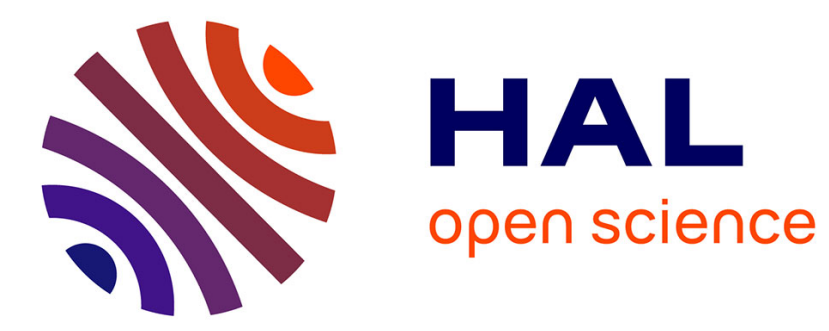

\title{
Unraveling the degradation of artificial amide bonds in nylon oligomer hydrolase: from induced-fit to acylation processes
}

Takeshi Baba, Mauro Boero, Katsumasa Kamiya, Hiroyuki Ando, Seiji Negoro, Masayoshi Nakano, Yasuteru Shigeta

\section{To cite this version:}

Takeshi Baba, Mauro Boero, Katsumasa Kamiya, Hiroyuki Ando, Seiji Negoro, et al.. Unraveling the degradation of artificial amide bonds in nylon oligomer hydrolase: from induced-fit to acylation processes. Physical Chemistry Chemical Physics, 2015, 17 (6), pp.4492-4504. 10.1039/C4CP04419C . hal-02991746

\section{HAL Id: hal-02991746 \\ https://hal.science/hal-02991746}

Submitted on 4 Dec 2020

HAL is a multi-disciplinary open access archive for the deposit and dissemination of scientific research documents, whether they are published or not. The documents may come from teaching and research institutions in France or abroad, or from public or private research centers.
L'archive ouverte pluridisciplinaire HAL, est destinée au dépôt et à la diffusion de documents scientifiques de niveau recherche, publiés ou non, émanant des établissements d'enseignement et de recherche français ou étrangers, des laboratoires publics ou privés. 


\title{
Unraveling the degradation of artificial amide bonds in Nylon oligomer hydrolase: From induced-fit to acylation processes
}

\author{
${ }^{*}$ Takeshi Baba ${ }^{\mathrm{a}, \mathrm{b}}$, Mauro Boero ${ }^{\mathrm{c}}$, Katsumasa Kamiya ${ }^{\mathrm{d}}$, Hiroyuki Ando ${ }^{\mathrm{a}}$, \\ Seiji Negoro ${ }^{\mathrm{e}}$, Masayoshi Nakano ${ }^{\mathrm{a}}$, ${ }^{*}$ Yasuteru Shigeta, ${ }^{\mathrm{f}, \mathrm{g}}$
}

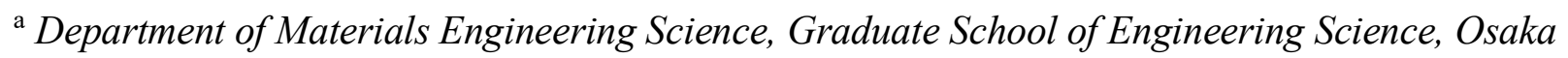
University, 1-3 Machikaneyama, Toyonaka, Osaka 560-8531, Japan.

${ }^{\mathrm{b}}$ Japan Society for the Promotion of Science (JSPS) Research Fellow.

${ }^{\mathrm{c}}$ Institut de Physique et Chimie des Matériaux de Strasbourg, UMR 7504 CNRS and University of Strasbourg, Strasbourg 23 rue du Loess, 67034 Strasbourg, France.

${ }^{\mathrm{d}}$ Center for Basic Education and Integrated Learning, Kanagawa Institute of Technology, 1030 Shimo-Ogino, Atsugi, Kanagawa 243-0292, Japan.

${ }^{\mathrm{e}}$ Department of Material Science and Chemistry, Graduate School of Engineering, University of Hyogo, 2167 Shosha, Himeji, Hyogo 671-2280, Japan.

${ }^{\mathrm{f}}$ Department of Physics, Graduate School of Pure and Applied Sciences, University of Tsukuba, 1-1-1 Tennodai, Tsukuba, Ibaraki 305-8571, Japan.

${ }^{\mathrm{g}}$ JST, CREST, 4-1-8 Honcho, Kawaguchi, Saitama 332-0012, Japan.

Corresponding authors: Takeshi Baba and Yasuteru Shigeta 


\begin{abstract}
To elucidate how the nylon oligomer hydrolase (NylB) acquires its peculiar degradation activity towards non-biological amide bonds, we inspect the underlying enzymatic processes going from the induced-fit upon substrate binding to acylation. Specifically, we investigate the mutational effects of two mutants, Y170F and D181G, indicated in former experiments as crucial systems because of their specific amino acid residues. To this aim, by resorting on first-principles molecular dynamics complemented with metadynamics we provide a detailed insight into the underlying acylation mechanism. Our results show that while in the wild type (WT) the Tyr170 residue points the NH group towards the proton-acceptor site of an artificial amide bond, hence ready to react, in the Y170F this does not occur. The reason is ascribed to the absence of Tyr170 in the mutant, replaced by phenylalanine, unable to form hydrogen bonds with the amide bond, thus resulting in an increase of the activation barrier of more than $10 \mathrm{kcal} / \mathrm{mol}$. Nonetheless, despite the lack of hydrogen bonding between the Y170F and the mutant, also in this case the highest free energy barrier for the induced-fit is similar to that of WT. This seems to suggest that, in the induced-fit process, kinetics is little affected by the mutation. On the basis of additional structural homology analyses on enzymes of the same family, we suggest that natural selection is responsible for the development of the peculiar hydrolytic activity of Arthrobacter sp. KI72.
\end{abstract}




\section{Introduction}

Nylon-6 is a synthetic fiber with a wealth of applications from textiles to electronics industry and, as such, increasingly produced by chemical companies worldwide. The fundamental reaction exploited is the ring-opening polymerization of $\varepsilon$-caprolactams. ${ }^{1,2}$ However, this has a non-negligible environmental impact, since it amounts to industrial-scale production of non-biological amide bonds in long fibers containing more than 100 units of 6-aminohexanoate (Ahx). In this respect, an unprecedented discovery dating back to the 1940's is the existence of Nylon-oligomer hydrolases, found in the Arthrobacter sp. KI72, able to degrade the nylon-6. This discovery brought to the attention of the scientific community that this bacterium was able to adapt to the presence of these non-biological compounds and, through natural selection, to develop a new functionality suitable to degrade the artificial Nylon-6 fibers at least for a relatively short period. Nowadays, three different types of the Nylon-oligomer hydrolases have been identified, namely Ahx-cyclic-dimer hydrolase (NylA), Ahx-liner-dimer (Ald) hydrolase (NylB), and endo-type Ahx-oligomer hydrolase $(\mathrm{NylC}){ }^{3-8}$ Each one of these hydrolase has its own inherent substrate specificity. Specifically, NylA degrades the amide bond present in a cyclic Ahx dimer to produce a 6-aminohexanoate-liner-dimer (Ald). NylB degrades the amide bond formed in a linear Ahx oligomer, especially Ald, from the N-terminal of the oligomer. NylC degrades amide bonds of both cyclic and linear oligomers. Moreover, NylC is also able to revert nylon- 6 into Ahx oligomers. ${ }^{7,8}$

Among these three Nylon-oligomer hydrolases, NylB has received special attention in a series of biochemical experiments. According to the reported genetic analyses, NylB has a closely resembling enzyme, NylB*, characterized by a $88 \%$ sequence identity. Nonetheless, despite this close similarity, the reactivity to the substrate (Ald) of $\mathrm{NylB}^{*}$ is lower than that of NylB by about a factor of $1 / 200 .^{3}$ In an attempt at enhancing the reactivity and to overcome the difficulty in their crystallization, hybrid enzymes of both $\mathrm{NylB}$ and $\mathrm{NylB}^{*}$ were made by replacing some amino acid residues in NylB ${ }^{*} .9$ This resulted in a set of 24 different structures labeled from Hyb-1 to Hyb-24 which were subsequently structurally analyzed by X-ray crystallography or crystallization. A noticeable example of this extensive investigation is represented by Hyb-24; this specific hybrid enzyme contains five amino acid residue substitutions with respect to the pristine $\mathrm{NylB}^{*}$ : T3A, P4R, 
T5S, S8Q, and D15G. Analogously, in the recently obtained Hyb-24DN and Hyb-24DNY a double mutation (G181D and $\mathrm{H} 266 \mathrm{~N})$ and a triple mutation (G181D, H266N, and D370Y) with respect to Hyb-24 were realized by one of us. ${ }^{10-13}$ The specific mutational sites were deduced by a comparison of the amino acid sequences of NylB and $\mathrm{NylB}^{*}$ (Asp181 and Asn266 in NylB, as opposed to Gly181 and His266 in NylB*). ${ }^{9,10}$ It is also worthy to note that polymerase chain reaction (PRC) applied in random mutagenesis experiments, accidentally resulted in the discovery of the D370Y mutation mentioned above; nonetheless, its role was a matter of debate. Only later ${ }^{12,13}$ kinetics analyses allowed to establish that the actual role of $\mathrm{D} 370 \mathrm{Y}$ is a stabilization of enzyme substrate complex. At the present state of knowledge, Hyb-24DNY possesses the highest activity among all the known mutants. ${ }^{12,13}$ For this reason, hereafter, we shall assume Hyb-24DNY as our reference system and indicate it as the "wild type" (WT) for simplicity. Judging from the similarities in the overall structure and the amino acid sequence, $\mathrm{NylB}$ belongs to the class $\mathrm{C}$ of the $\beta$-lactamase family, which is characterized by the presence of a catalytic triad, Ser, Tyr, and Lys, having the ability to promote the hydrolysis of amide and/or ester bonds. ${ }^{9}$ According to X-ray crystallographic analyses, ${ }^{11,13}$ the WT can assume two different conformations, (i) a substrate-free form (open form) and (ii) a substrate-bound form (closed from) (see Fig.1 (a) for details). At variance with the other enzymes of the same family, the distinctive feature of NylB is the presence of a unique amino acid residue, Tyr170, near the catalytic triad, which forms a hydrogen bond (H-bond) with Ald, only in the closed form as sketched in Fig. 1(b).

Site-directed mutagenesis investigations were conducted on Hyb-24DN, the mutant in which the Tyr170 residue is replaced by a phenylalanine. These studies revealed that the hydrolytic activity of Ald is nearly suppressed, becoming only about $1.4 \%$, and its kinetics parameter values ${ }^{14,15}$, namely the Michaelis constant $\left(K_{\mathrm{M}}\right)$ and the rate constant $\left(k_{\text {cat }}\right)$ could not be determined because of such a low activity. ${ }^{11}$ This is an additional difficulty with respect to other mutants such as, for instance, Hyb-24Y (one mutation of D370Y from Hyb-24), Hyb-24DY (double mutation of G181D and D370Y from Hyb-24), Hyb-24DN, and Hyb-24DNY, for which the relevant kinetic parameters could be determined. ${ }^{13}$ This can again be ascribed to the fact that the Tyr 170 residue is present both in Hyb-24 (NylB* type) and in the WT (NylB type). On these grounds, it can be inferred that Tyr170 
is a crucial actor in the hydrolytic activity promoting the cleavage of non-biological amide bonds. Yet, the atomic-level elucidation of the underlying mechanism and the actual role of Tyr170 present still unclear issues. Part of these unresolved points could be recently addressed by our group ${ }^{16}$ by resorting on hybrid quantum mechanics / molecular mechanics (QM/MM) dynamical simulations complemented with free energy sampling methods, allowing to unravel the reaction mechanism in NylB and addressing one of the possible roles of Tyr170 during the acylation process. The latter is the rate limiting step of the series of enzymatic reactions operated by NylB. We also noticed that the residue Asp181 does not contribute directly to the acylation process, because of its relatively distance from the active site (Fig. 1 (c)). Despite being a considerable step forward in the understanding of the uncommon enzymatic activity of NylB, several points remain obscure and a comprehensive picture of the whole mechanism leading to the degradation of amide bonds of non-biological origin has still to be reached. Indeed, several processes are involved, which can be schematically summarized in four principal steps: (1) substrate binding, (2) induced-fit, (3) catalytic (enzymatic) reaction, and (4) final detachment of the substrate. Since the introduction of mutations is expected to affect several processes at the same time, it is necessary to analyze at least the steps (2) and (3), representing the main core of the overall reaction and involving directly the amino acid residues present in the pristine system or substituted upon the mutations. What is left out in the present study are then the initial docking (1) and the final release of the substrate (4), which, however, do not involve chemical reactions. On the other hand, we focus here (i) on the induced-fit of a specific loop region in NylB to the substrate and (ii) on the acylation processes of both the WT and the Y170F mutant. Our purpose is to shed some light into the peculiar hydrolytic activity of NylB and to address the open question about the absence of such an activity in other peptidases and lactamases. Coming to the issue of the docking of the enzyme onto the substrate, we pay special attention here to the mutational effects of D181G on the stabilization of the enzyme-substrate binding aimed at the enhancement of the substrate specificity in comparison with NylB and NylB*.

This paper is organized as follows. In section 2 we review the computational approaches used for the reactive QM/MM Car-Parrinello dynamics (CPMD), ${ }^{17,18}$ for the Parallel Cascade Selection MD (PaCS-MD) scheme, ${ }^{19,20}$ and for the inter-fragment interaction energy (IFIE) analyses based on 
the fragment molecular orbital (FMO) method. ${ }^{21-23}$ In the Section 3, we discuss in detail the results of each simulation performed and, on the basis of structural homology analyses, we propose a computationally supported answer to the question concerning the origin of the hydrolytic activity of NylB. Finally, in Sec. 4 we draw our conclusions.

Fig. 1

\section{Computational details}

\subsection{Preparation of target systems}

We started the preparation of the prototype structure for the WT enzyme-substrate complex from the X-ray coordinates available at the Protein Data Bank (PDB entry: 2zma). In this system the Ser112 residue is replaced by Ala to crystallize the complex. To revert back to the system targeted in this study, we replaced Ala112 with the original Ser 112 residue and added all the missing amino acid residues to obtain an appropriate WT model. Analogously, the structure of the Y170F mutant was reconstructed by replacing Tyr170 with Phe170 in the WT modeled as explained above, whereas the Hyb-24 was obtained by substituting Asp181, Asn266, and Tyr370 with Gly181, His266, and Asp370 in WT, respectively. The target WT system used in the simulations consists of

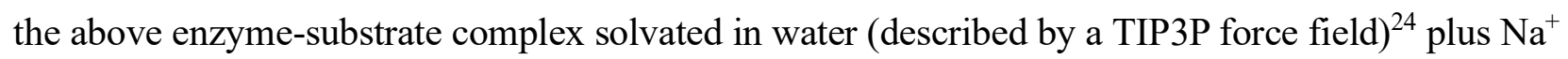
counter-ions to neutralize the system; such a model amounts to 41403 atoms.

The system was prepared by first equilibrating the solvated complex via classical molecular dynamics (MD) for about 10ns in an NPT ensemble, by setting the target temperature and pressure at $300 \mathrm{~K}$ and $1 \mathrm{~atm}$, respectively. To this aim, the PMEMD module (SPFP precision model) of the AMBER $^{25,26}$ program suite was used. The force field adopted for the amino acid residues was a standard Amber ff99, while for Ald the force field was generated ad hoc with the AMBER GAFF tool (see Ref. 27) for details using the Antechamber program of the AMBER package. ${ }^{25}$ The solvated system is placed in a simulation cell of $77.57 \times 76.62 \times 69.30 \AA^{3}$ where periodic boundary condition are applied and the SHAKE algorithm ${ }^{28}$ was used all across MD simulations to satisfy bond geometry constraints. The long-range interactions are evaluated by means of the particle mesh Ewald method with a cutoff of $12.0 \AA .^{29}$ The protonation states adopted here for each amino acid 
residue are the ones originally determined by the group of J. H. Jensen ${ }^{30-33}$ and partly the ones deduced by direct inspection of their local hydrogen bonding environments. In particular, Lys115, which is one of the fundamental amino acid residues in the active site, was set to be deprotonated $\left(-\mathrm{NH}_{2}\right)$ to promote the hydrolysis.

\subsection{Reactive QM/MM Car-Parrinello simulations for the acylation}

The acylation reaction, which involves cleavage and formation of chemical bonds, was simulating within a reactive hybrid QM/MM approach ${ }^{34,35}$ complemented with metadynamics $^{36-40}$ in which the QM driver is the Car-Parrinello molecular dynamics (CPMD) ${ }^{41}$ based on the density functional theory. ${ }^{42,43}$ The aim is the investigation of the reaction mechanism of the Y170F mutant and the estimation of the free energy difference between WT and Y170F. This approach has already been extensively used and benchmarked many biological systems. ${ }^{44-51}$ In our specific systems, the embedded QM region is composed of 103 atoms in the case of the WT and 102 atoms in the case of the Y170F mutant (Fig. 1 b). The total net charge for the QM subsystem equal to $-1 e$ and monovalent hydrogen-like link atoms are used to saturate dangling bonds at the interface between the QM and the MM regions. Further details concerning the QM subsystems can be found in the supporting information (SI-1). The CPMD package ${ }^{52}$ was used for all these simulations. The exchange correlation functional used here is the one proposed by Hamprecht, Cohen Tozer and Handy $(\mathrm{HCTH}),{ }^{53}$ the core-valence interaction is described by Martins-Troullier pseudopotentials ${ }^{54}$ and valence electrons are represented in a plane wave basis set with an energy cut off of 70 Ry. All QM/MM CPMD simulations were done in a canonical NVT ensemble at a target temperature of $300 \mathrm{~K}$. The fictitious electron mass for the integration of the CPMD electronic equations of motion was set to 400 a.u. and the time step adopted was 4 a.u. (0.096 fs). Classical MM interactions were described by an Amber ff99 force field. Before moving to the reactive metadynamics ${ }^{36,37}$ simulations, the system was equilibrated via classical MD in an NVT ensemble set-up for $1 \mathrm{~ns}$, then, subsequently, and unbiased QM/MM CPMD dynamics of the same type was performed to equilibrate also the newly added electronic degrees of freedom for about 2 ps. After the equilibration, metadynamics simulations were done with the purpose of understanding whether or 
not the role of the catalytic triad Ser112-Lys115-Tyr215 is identical both in the WT and in the mutant. Although the reaction mechanism for the WT has already been reported elsewhere, ${ }^{16}$ we repeated this simulation using one single collective variable as explained later. On one hand, this provides a further and independent check of our former results and, on the other hand, the identical scheme applied to the Y170F mutant allows for a direct comparison of the relative reactivity of the two systems. To the benefit of the explanation of the parameters adopted, we recall that, in its Lagrangean formulation, the metadynamics scheme is summarized by the extension of the Car-Parrinello Lagrangean $L^{\mathrm{CP}}$ to include the selected reaction coordinates, referred to as collective variables (CVs) hereafter, namely

$$
L=L^{\mathrm{CP}}+\frac{1}{2} \sum_{\alpha} M_{\alpha} \dot{s}_{\alpha}^{2}-\frac{1}{2} \sum_{\alpha} k_{\alpha}\left[S_{\alpha}\left(\left\{\mathbf{R}_{I}\right\}\right)-s_{\alpha}\right]^{2}+V\left(t, s_{\alpha}\right)
$$

Concerning the single $\mathrm{CV}, s_{1}$, used in the present case, we selected the distance between $\mathrm{O}_{\gamma}$ atom (OG) of Ser112 and carbonyl carbon (C1) of Ald393 (indicated by a green arrow in Fig. 1 c), which properly represents the nucleophilic attack of Ser112 to the amide carbon present in the substrate. The history dependent potential $V\left(t, s_{\alpha}\right)$ was constructed by accumulation of small Gaussian penalty functions having height width of $0.5 \mathrm{kcal} / \mathrm{mol}$ and 0.3 au and a new Gaussian function was added every 140 steps (13.44 fs), which will be referred to as one meta-step hereafter (Details of other parameters are shown in SI-2). The history-dependent term in principle converges asymptotically to the free energy surface (FES) $F\left(s_{\alpha}\right)$ which can then be computed as the average

$$
F\left(s_{\alpha}\right)=-\frac{1}{t_{\text {simul }}-t_{\text {diff }}} \int_{t_{\text {diff }}}^{t_{\text {simul }}} V\left(s_{\alpha}, t\right) d t,
$$

to reduce statistical errors ${ }^{55}, t_{\text {simul }}$ being the total simulation time and $t_{\text {diff }}$ the time at which CVs start diffusing in all the sampled phase space. For the present purpose, this can be assumed to be the completion of the acylation. Indeed, in this case, we followed the simulation until the first reaction occurs, since we focus specifically on the relative free energy barriers in WT and Y170F for this specific process. Because of the specific choice of collective variable, the system is unable to realize the reverse reaction unless reflecting walls able to reverse the momenta of the leaving atoms are 
used (as e.g. in Ref. 45). Since this would result in a lack of momentum conservation, we avoided it to prevent any further biasing. However, this procedure is sufficient to estimate, at least approximately, free energy differences. Results were analyzed with our own program and visualized by $\mathrm{VMD}^{56}$

\subsection{PaCS-MD simulation for induced-fit process}

To investigate the difference between the WT and the Y170F mutant in the induced-fit processes, we resorted on the PaCS-MD approach, which generates reactive trajectories from a reactant (open form) to a product (closed form) by combining a series of short-time simulations. ${ }^{19,20}$ Each one of the two structures was extracted from a 10 ns classical MD simulation. The induced-fit mechanism concerning the WT has already been discussed in a former work ${ }^{20}$ and we refer to those results for the ongoing discussion. Instead, for the Y170F mutant PaCS-MD was done for 25 cycles starting from 10 different initial configurations sampled on the basis of the same selection rule formerly adopted, specifically the root mean square displacement (RMSD) of the loop-segment (Asn166-Val177) except for all hydrogen atoms, invisible to X-ray crystallographic inspection, and $\mathrm{C}_{\alpha}$ atoms of the $111^{\text {th }}$ to $116^{\text {th }}$ residues. This specific selection rule has been shown to efficiently trigger the induced-fit for $\mathrm{WT}^{20}$ To evaluate the free energy change due to the induced-fit, we adopted the weighted histogram analysis method (WHAM) ${ }^{58}$, where 2000 reference structures (200 frames randomly selected selection in each one of 10 independent reactive trajectories) were used for the WHAM inspection. A biased umbrella potential with a coupling parameter $k=0.5 \mathrm{kcal} / \mathrm{mol}$ $\AA^{2}$ was imposed on the loop-segment, apart from $\mathrm{H}$ atoms, the nitrogen atom of the amide bond of Ald, and the $\mathrm{C}_{\alpha}$ atoms of the $111^{\text {th }}$ to $116^{\text {th }}$ residues. The free energy obtained in this way was then projected onto two specific distances: The separation between $\mathrm{C}_{\delta}$ atom (CD) of Glu168 and $\mathrm{C}_{\alpha}$ atom $(\mathrm{CA})$ of Ser217, $d_{1}$, and the one between $\mathrm{H}_{\zeta}$ atom $(\mathrm{HH})$ of Phe170 (replacing $\mathrm{O}_{\zeta}$ atom $(\mathrm{OH})$ of Tyr170 in the WT) and nitrogen atom in amide bond (N3) of Ald393, $d_{2}$, which clearly correspond to the reaction coordinates previously adopted for the WT. ${ }^{20}$

\subsection{FMO calculations of enzyme substrate bound state of Hyb-24 and Y170F}


To better quantify the interaction between ALD and the specific amino acid residues involved, namely Asp181 in Hyb-24 and Tyr170 in the Y170F mutant, we made use of an inter-fragment interaction energy (IFIE) analyses based on the fragment molecular orbital (FMO) method. ${ }^{21}$ The total energy, $E$, in FMO framework is expressed as

$$
E=\sum_{I} E_{I}-\sum_{I>J}\left(E_{I J}-E_{I}-E_{J}\right)
$$

where $E_{I}$ and $E_{I J}$ represent the fragment energies of $I$-th monomer and $I J$-th dimer, respectively. ${ }^{53}$ The IFIE value is defined as ${ }^{59}$

$$
\Delta E_{I J}=E_{I J}^{\prime}-E_{I}^{\prime}-E_{J}^{\prime}+\operatorname{Tr}\left[\Delta \mathbf{P}_{I J} \mathbf{V}_{I J}\right]
$$

where $\Delta \mathbf{P}_{I J}$ is the matrix constructed by computing the difference between the $I J$-th dimer and two $I$-th and $J$-th monomers, while $\mathbf{V}_{I J}$ is the environmental electrostatic potential of the $I J$-th dimer with respect to all the other remaining fragments. The superscript on $E$ ' indicates the bare fragment energy without the inclusion of the environmental electrostatic potential.

All FMO calculations were carried out as implemented in the ABINIT-MP program suite ${ }^{60}$ at the FMO2 MP2/6-31G(d) level. To reduce the computational costs, we limited the calculations to one single snapshot extracted from a 10 ns trajectory obtained upon classical MD. Moreover, explicit solvent effect on the stability of the enzyme-substrate bound form was accounted for by the inclusion of all water molecules included in a region of $15 \AA$ around the substrate. ${ }^{61,62}$ The results obtained by IFIE were visualized with the BioStation Viewer. ${ }^{63}$

\section{Results \&Discussion}

Fig.2

\section{$\underline{\text { Acvlation process }}$}

The starting point of our analysis is the acylation process of the Y170F simulated via reactive QM/MM CPMD metadynamics. The changes occurring in the chemical bonds upon acylation as provided by the metadynamics simulation are sketched in Figure 2. The specific bonds to which we refer (shown in Fig.1 (c)) are $r_{1}$ : N3(Ald393)-C1(Ald393), $r_{2}$ : OG(Ser112)-C1(Ald393), $r_{3}: \mathrm{HH}(\mathrm{Tyr} 215)-\mathrm{N} 3(\mathrm{Ald} 393)$, and $r_{4}: \mathrm{H}_{\gamma}$ atom (HG) (Ser112)- $\mathrm{N}_{\zeta}$ atom (NZ)(Lys115). More 
precisely, $r_{1}$ represents the amide bond undergoing cleavage and dissociation at the end of the acylation reaction, $r_{2}$ and $r_{3}$ represent the distance of the nucleophile from the substrate and that from the donor proton to the substrate, respectively. Both these distances shrink during the process until they become proper chemical bonds once the acylation is realized. The change in $r_{4}$ is useful to monitor the nucleophilic character whenever a proton transfer attempt to the base happens during the process. In fact, on the basis of the variations of $r_{4}$, we noticed that at three different stages along the reactive trajectory a nucleophilic attack of Ser112 occurs (dotted circle regions in Fig. 2-(a)). Eventually, at the third and last attempt, the acylation occurred (meta-step 1240) as easily inferred from the changes in $r_{1}$ and $r_{3}$. In order to get a better insight into the failure of the former two attempts, which did not result in the acylation, we inspected the configurations visited by the reactive trajectory schematically shown in Figs. 2 (b) and (c). Comparing (b) and (c) with (d), we noticed a change in $r_{3}$ of different nature with respect to the one realized in the actual final acylation. At the same time, $r_{1}$ keeps constantly a distance close to the regular amide bond without any sign of destabilization. This is deeply different from the case of Fig. 2 (d), where a hydrogen atom transfer from Ser112 to Lys115 ( $\left.r_{4} \sim 1.0 \AA\right)$ is realized and the nucleophilic attack of Ser112 can occur resulting in the formation of a chemical bond with the carbonyl carbon of the amide bond of Ald $\left(r_{2} \sim 1.2 \AA\right)$. Accompanying these processes, the amide bond of Ald immediately dissociated after Tyr215 protonates the nitrogen atom of the amide bond $\left(r_{3} \sim 1.0 \AA\right)$. Such a reaction mechanism for the Y170F mutant is consistent with that of $\mathrm{WT},{ }^{16}$ (see SI-3) and the estimated free energy barrier is reported in Table 1. We recall that a typical error bar of $1-2 \mathrm{kcal} / \mathrm{mol}$ affects these values. Errors can be kept under control in the variational formulation of metadynamics used in our approach, which allows collective variables to oscillate around the "exact" solution in a way identical to the one in which the Car-Parrinello fictitious electron kinetic energy fluctuates around the exact BO trajectory. The dynamics of these extra degrees of freedom can be explicitly controlled by tuning the fictitious mass (kinetic term) and spring constant (harmonic term) and/or by suitable thermostats ${ }^{55}$ and, thus, can be smoothened. Nothing, instead, can be done for the underlying DFT level. ${ }^{83}$

The most remarkable feature is the larger value of this barrier for the Y170F mutant with respect to the WT, namely $11 \mathrm{kcal} / \mathrm{mol}$ above the analogous barrier for the $\mathrm{WT}^{16}{ }^{16}$ in qualitatively good 
agreement with experiments in which mutations of Y170F resulted in a dramatic decrease in the Ald degradation activity to about $1.4 \%$ with respect to WT. ${ }^{11}$

\section{$\underline{\text { Table } 1}$}

\section{Fig. 3}

To elucidate the differences between the WT and its Y170F mutant during the acylation, the structural changes, especially when Tyr215 provides the $\mathrm{H}$ atom to the amide $\mathrm{N}$ atom of the substrate, we compared the evolution of the mutant with respect to the WT system as sketched in the panels of Fig. 3. Before the acylation, the amide bond and the neighboring atoms are all located on an amide plane. Then, when the acylation occurs, the $\mathrm{O}$ atom belonging to the side chain of Ser112 attacks the amide bond forming an $\mathrm{sp}^{3}$ bonding configuration which includes the amide carbonyl $\mathrm{C}$ atom, resulting in the breaking of the initial planar symmetry mentioned above. Simultaneously, the amide $\mathrm{N}$ atom also assumes an $\mathrm{sp}^{3}$ geometry. During this process, in the case of the WT, the Tyr170 contributes to the reaction with its own $-\mathrm{OH}$ group: This $-\mathrm{OH}$ forms one H-bond with the $-\mathrm{NH}$ moiety of the amide bond of Ald and this, in turn, forces the nitrogen atom in Ald to keep its lone pair oriented toward Tyr215. In such a configuration, the amide $\mathrm{N}$ pointing toward Tyr215 is ready to accept the $\mathrm{H}$ atom from Tyr215, as confirmed by the formation of a new and tight $\mathrm{H}$-bond between the phenol $\mathrm{OH}$ and the amide NH group. At variance with the WT case, in the Y170F mutant (Fig. 3-(b)) the absence of Tyr170 and its H-bond does not allow the amide $\mathrm{NH}$ group to assume a configuration suitable to accept the $\mathrm{H}$ atom from Tyr215, thus suppressing the amide bond cleavage activity. This picture is perfectly consistent with the fact, recently demonstrated $^{64}$, that the specific orientation of the lone pair of the nitrogen of the scissile amide bond during catalysis is a crucial feature of enzyme catalyzed amide bond hydrolysis. After a few additional meta-steps, a water molecule from the surrounding solvent approaches to form an H-bond with the amide NH group as shown in Fig 3-(c), thus replacing the role of Tyr170 in the WT. From the point of view of the local H-bond environment only, this conformation resembles closely the one of the WT shown (Fig3-(d)). In addition, it is expected that hydrogen bond might contribute the acceptance tendency of hydrogen atom from Tyr2 15 as well as the control of direction of NH group considering the Fig. 3 (a). This indicates rather unambiguously that the formation of an 
H-bond to the amide $\mathrm{NH}$ group is indispensable to trigger the $\mathrm{H}$ transfer from Tyr215 and that Tyr170 provides the fine control needed to keep the local geometry in a condition suitable to give rise to an efficient breaking of the amide bond. We can then infer that Tyr170 is the fundamental additional residue that must complement the catalytic triad to realize the acylation in this enzyme. Its absence, as in the case of the mutant, results clearly in the suppression of this specific activity or in a larger activation barrier that makes acylation highly improbable. For amines, two different $\mathrm{sp}^{3}$ configurations are in general allowed, as shown in Fig. 3-(e), and the conformational change between them is well known as an umbrella inversion, whose barrier is about $6 \mathrm{kcal} / \mathrm{mol}$ for an ammonia molecule in the gas phase. ${ }^{65}$ By reasoning on these grounds, the order of magnitude of the free energy difference between WT and the Y170F mutant in terms of activation barrier $(\sim 11$ $\mathrm{kcal} / \mathrm{mol}$, according to our estimates) might be compatible with the results obtained via the umbrella inversion technique also in a protein environment.

Comparing our result with theoretical studies on general serine proteases, ${ }^{66-68}$ structures like the one reported in Fig. 3-(e) were found, for instance, in Fig. 4 of Ref. 62 and discussed in the context of the stereochemistry of tetrahedral intermediates in Ref. 61. In the typical proteases, such as, for instance, chymotrypsin and trypsin, a substrate pocket able to bind to their specific substrate is always present. In the substrate pocket, the chymotrypsin and the trypsin recognize bulky aromatics and side chain in a peptide, respectively, enabling them to identify the site to be cleaved. Instead, the substrate-binding site of NylB is more like a cleft, and in principle can bind substrates so weakly that simple mechanical fluctuations in substrate and changes in the organization of water molecules around the substrates result in a decrease of the reactivity. Only after the induced-fit of Tyr170, allowing to smooth the fluctuations of the substrate in the vicinity of the catalytic triad and to prevent approaching of water molecules during the acylation phase, NylB can degrade the Nylon-oligomers.

So far, focusing on the acylation processes, we established the different behavior of the WT and the Y170F mutant, providing a solid argument for the decrease in the hydrolytic activity of the latter. Nonetheless, a still unaddressed question is the actual role of the Tyr170 residue in the induced-fit to bind the substrate. 


\section{Induced-fit process}

To complete the picture about the overall role of Tyr170, we investigated the induce-fit process. To this aim, the free energy surface (FES), projected onto two reaction coordinates $d_{1}$ and $d_{2}$, was estimated by WHAM making use of a set of reactive trajectories generated via PaCS-MD simulations (Fig. 4 (a)). This analysis has shown that four minima (A)-(D) are located on this FES, where the distances in the reference structures are $\left(d_{1}, d_{2}\right)=(10.89 \AA, 14.11 \AA)$ for the open-form and $\left(d_{1}, d_{2}\right)=(4.75 \AA, 4.05 \AA)$ for the closed-form, respectively. As shown in Fig. 4 (a), starting from the open-form, both distances decrease from $\left(d_{1}, d_{2}\right)=(10 \AA, 14 \AA)$ in the basin $\mathrm{A}$ to $\left(d_{1}, d_{2}\right)=(9.0 \AA$, $7.5 \AA$ ) in the next local minimum B. In particular, the decrease along the $d_{2}$ direction is relatively larger than that along $d_{1}$. Subsequently, the system reaches the minimum $\mathrm{C}$ at $\left(d_{1}, d_{2}\right)=(7.1 \AA, 5.5 \AA)$, while $d_{2}$ decreases almost monotonically to a value close to the closed-form. From $\mathrm{C}$ to $\mathrm{D}$, characterized by distances $\left(d_{1}, d_{2}\right)=(4.8 \AA, 5.2 \AA), d_{1}$ undergoes a noticeable decrease, whereas $d_{2}$ remains almost constant. This point (D) is the global minimum of our FES landscape. Yet, the free energy difference between (C) and (D) is only $\sim 0.2 \mathrm{kcal} / \mathrm{mol}$, on the verge of the accuracy of these numerical methods, while the other minima are located at slightly higher values, which however are of the order of magnitude of $1 \mathrm{kcal} / \mathrm{mol}$. These shallow minima separated by tiny barriers indicate that the loop region might fluctuate among them. The stabilization energy due to the induced-fit can be as the free energy difference between (A) and (D) and turns out to be (A) - (D) $=0.6 \mathrm{kcal} / \mathrm{mol}$. The highest free energy barrier is the one separating (C) from (D) and its value is about $2.4 \mathrm{kcal} / \mathrm{mol}$. Additional information can be found in the supporting information (SI-4).

Fig.4

To elucidate the mutational effects of Y170F on the induced-fit process (Fig. 4 (a)), we compared the present results with the previous ones for the $\mathrm{WT}^{20}$ Focusing on the FES landscape of both systems, two major features are worthy of note. The first one is the number of (meta-) stable structures realized by the two systems, namely six in the case of the WT, as opposed to four for the Y170F mutant. The second one is the size of each basin where these local minima are located. In the case of the Y170F mutant, the areas of the free energy landscape around the minima, especially 
(A) and (B), but also the other remaining two, are rather wide and characterized with not very steep slopes with respect to the WT. Although Glu168 is a residue common to both the WT and the mutant, in the first case it forms an H-bond with the substrate and Ser217; conversely fluctuation of Phe170 in the Y170F mutant prevents Glu168 from forming any H-bond with Ser217. Despite this important structural difference, the highest free energy difference in the FES of the Y170F mutant is nearly identical to that of the WT. These findings indicate that the mutational (structural) effect due to the induced-fit on the FES landscape is remarkable, while the stabilization energy is small from a kinetic point of view.

\section{Substrate binding stability of Hyb-24 and Y170F mutants}

In an attempt at quantifying the effect of each residue on the overall stability of the enzyme-substrate bound form, such as Hyb-24, in particular the so-called D181G mutant, and the Y170F mutant, we performed IFIE analyses based on the FMO method. More precisely, we focused on the IFIE values between each ALD fragment and the most relevant amino acid residues, such as Tyr170 and Asp181 (see Fig. 5). In order to analyze contributions of IFIE in more detail, we further divided the substrate into three distinct fragments: the C-terminal (Ald(1)), the amide group (Ald(2)), and the N-terminal(Ald(3)), as shown in Fig 5. The result of this analysis is summarized in Table2. The IFIE values for the WT have been already elsewhere ${ }^{56}$ and shown in SI-5. According to Table 2, Asp 181 is characterized by an attractive interaction with Ald(3) stronger than that of Tyr170 with Ald(2). Conversely, in Hyb-24 the interaction between NylB and $\operatorname{ALD}(3)$ is lacking. Although the IFIE value to Ald(2) of Phe170 in the Y170F mutant becomes weaker than that of Tyr170 in the WT, the decrease of the IFIE is less pronounced than that to Ald(3) of the Hyb-24. These results suggest that Asp181 is quantitatively, energetically speaking, an amino acid residue more important than Tyr 170 for the specific substrate binding. Indeed, available kinetics data on Hyb-24DY, resulting from a double mutation of G181D and D370Y from Hyb-24, $\left(k_{\text {cat }} / K_{\mathrm{M}}=0.35 \mathrm{~s}^{-1} \mathrm{mM}^{-1}, K_{\mathrm{M}}=7.1 \pm 1.28 \mathrm{mM}^{-1}\right)$ and of Hyb-24Y, in which a single mutation of D370Y from Hyb-24 is present, $\left(k_{\text {cat }} / K_{\mathrm{M}}=0.016 \mathrm{~s}^{-1} \mathrm{nM}^{-1}, \quad K_{\mathrm{M}}=39.1 \pm 6.73 \mathrm{mM}^{-1}\right),{ }^{13}$ indicate rather clearly that Asp181 contributes to enhance the substrate specificity. 


\section{Homology analyses on NvIB and possible molecular evolution route for Ald hydrolvtic activity}

Our computational results, corroborated by experiments, suggest that, from the evolutional point of view, the introduction of Y170 in the loop region is at the origin of the acquisition of the hydrolytic activity from NylB. To bring support to our assumption, we completed our study by investigating any homologous enzyme exists possessing a similar evolution for the substrate binding. To this purpose, we performed structural homology searches for the WT (PDB id: $2 \mathrm{zma}$ ) by using the DALI program ${ }^{69}$ and selected structures preserving the local configuration around Tyr170 similar to NylB. This search, performed by using MultiSeq2.0 with the STAMP algorithm. ${ }^{70-72}$, brought to our attention the EstU1 system, having a high homology to NylB, as shown in Fig. 6 (a). The specific substrate of EstU1 is a cephalothin, which has bulky functional groups. Around the binding site of the substrate, EstU1 presents three amino acid residues, Tyr172, Phe174, and Phe190, which correspond to Glu168, Tyr170, and Asp181 in NylB, respectively. These three aromatic amino acid residues recognize the thiophene ring of cephalothin cooperatively by hydrophobic interactions (see Fig. 6 (b)). The remarkable difference between $\mathrm{NylB}$ and EstU1 is the location of the helix structure and the loop-segment evidenced in Fig. 6. In addition to the similarity in the structure, the side chain of Phe174 in the loop-segment of EstU1 undergoes a conformational change during the substrate binding process. This property is resembles closely the one of NylB. We can then infer that, upon acquiring Tyr170, NylB might have undergone a natural selection process to adapt to a nylon-oligomer-rich environment by skillfully using the loop region for the induced-fit to the substrate and by catalyzing the acylation via a decrease if the activation barrier.

\section{Fig.6}

\section{Towards a rational desion of enzymatic activities}

Having unraveled the role of Tyr170 in the WT, which contributes mainly to the acylation step to regulate the direction of a hydrogen bond, we have now a convincing set of results allowing to proceed further. All our results concur to support the notion that $\mathrm{NylB}^{*}$ needs not only the catalytic triad Ser-Tyr-Lys, like the class C $\beta$-lactamase family, but also Tyr170 to degrade non-biological amide bonds present in nylon-oligomers. In particular, acquiring Tyr170 in NylB ${ }^{*}$ is the key factor for 
triggering the reaction by decreasing the activation barrier, in other words by decreasing the rate constant $k_{\text {cat }}$. This is confirmed by a comparison with Hyb-24 (nearly identical to NylB* ${ }^{*}$, which possesses as well a weak reactivity to Ald. In the change from $\mathrm{NylB}^{*}$ to NylB, Asp181 provides the required substrate specificity, resulting in the decrease of $k_{\text {cat }}$.

Recent advances in computer facilities and related theoretical methods allow nowadays for a de novo design of functional proteins. ${ }^{73}$ In the last decade, researchers working in this field have succeeded in producing new enzymes called "theozymes", which were entirely designed on a computer by inserting a suitably selected transition state structure into protein scaffolds. ${ }^{74-77}$ Unfortunately, for these theozymes, the resulting catalytic proficiency, defined as

$$
\text { Catalytic proficiency }=\frac{\left(k_{\text {cat }} / K_{\mathrm{M}}\right)}{k_{\text {uncat }}} \quad\left(\text { in } \mathrm{M}^{-1}\right) \text {, }
$$

was still inferior to the natural one. In this expression $k_{\text {uncat }}$ is the rate constant in the absence of the enzyme, ${ }^{78}$ while $k_{\text {cat }}$ is the rate constant of the theozyme, lower than the natural one. ${ }^{79}$ The lesson to be retained is that looking just at the structure of the building block (transition state in this case) to be inserted inside a given scaffold is insufficient to obtain an enhancement of the catalytic activity. In this respect, in our study we have shown that Tyr170 and Asp 181 play an important role in direct and indirect decrease of $k_{\text {cat }}$ by stabilizing both the transition state and the substrate binding structure, thus allowing for an efficient degradation of the non-biological amide bond. Compared with the strategy followed for the theozyme, the change realized in the passage from Hyb-24 (NylB* type) to WT or Y170F (NylB type) could resemble the processes of the transition state design and the active site re-design briefly summarized above. Nonetheless, the theozyme was designed and realized in a laboratory and never underwent any natural selection process. Its absence in nature might be ascribed to its relative rigidity, which, contrary to other enzymes, implies a lack of dynamic motions of the flexible loop regions. We can then expect that taking into account also the response of the system to natural selection, as in the case of $\mathrm{NylB}$, can be the key for a rational design of enzymatic reactions with appreciable catalytic proficiency, although we have to acknowledge that a major difficulty is still represented by the prediction of the possible conformational changes an enzyme could assume. ${ }^{80}$ 
Some hints could be obtained by the development of integrated schemes making use of coarse grained $\mathrm{MD}^{81}$ or Fluctuation Flooding Method ${ }^{82}$ jointly with quantum chemical transition state design not too far from the strategy adopted for the theozyme.

\section{Conclusions}

A combination of different theoretical methods was used to inspect in detail a series of enzymatic reaction representing the fundamental stages in the degradation of non-biological amide bonds operated by the nylon oligomer hydrolase NylB. To this aim a comparative study of the WT enzyme and its closely related mutant was conducted, unveiling the crucial role of two evolutionally important amino acid residues, Tyr170 and Asp181, in (1) the enzymatic reactions, (2) the induced-fit process, and (3) the stability of the enzyme-substrate binding. We found at which steps of this complex process Tyr170 and Asp181 are involved and which are their respective net effects on the overall process. Specifically, Tyr170 mainly contributes to the acylation step and plays a subsidiary role in the stabilization of the enzyme-substrate complex. According to the free energy changes during the acylation and the induced-fit steps upon the mutation, in the mutant the former process requires the overcoming of an activation barrier which is $11 \mathrm{kcal} / \mathrm{mol}$ higher with respect to the WT case. This is clearly a stumbling block to acylation that makes the reaction very unlikely for the Y170F system. Conversely, for the induced-fit process of the Y170F mutant, although the free energy landscape changes drastically with respect to the WT case, the free energy barrier is nearly unchanged, suggesting that the kinetics of the induced-fit is barely affected by the mutation or, at least, this mutation. Although the inter-fragment interaction energy (IFIE) of Phe170 in the Y170F mutant is lower than that of Try170 in the WT, such a decrement is smaller than that of Hyb-24. Based on all these results, we can conclude that Tyr170 is the essential crucial amino acid residue to promote the degradation of non-biological amide bonds by forming a novel type of a catalytic quad, as opposed to standard catalytic triads, responsible for both the stabilization of the transition state and the restraining of fluctuations in the substrate binding structure. On the other hand, Asp181 mainly contributes to the stabilization of the enzyme-substrate complex (substrate specificity), while the influence on the hydrolytic activity is negligible. 


\section{ACKNOWLEDGMENT}

We thank Mr. Masanari Matsuoka for insightful discussions in the search for structural homology. This work is supported by Grant-in-Aid for Scientific Research of the Innovative Areas "Computics", "Dynamic ordering and biofunction", and Photosynergetics" (No. 25104716, 26102525, and 26107004), and a Grant-in-Aid for Young Scientists (B) (Grant No. 22740259) from Japan Society for the Promotion of Science (JSPS), and by the Strategic Programs for Innovative Research (SPIRE), MEXT, and the Computational Materials Science Initiative (CMSI), Japan. T.B. expresses his special thanks to the JSPS Research Fellowship for Young Scientists (No. 02503029). Theoretical calculations were partly performed at the Research Center for Computational Science, Institute for Molecular Science (Japan) and Center of Computational Materials Science, Institute for Solid State Physics, The University of Tokyo, (Japan). M.B. and T.B. acknowledge the Direction Informatique (Pôle HPC) of the University of Strasbourg for providing scientific support and access to computing resources. Part of the computing resources were funded by the Equipex Equip@Meso project. K.K. acknowledges the Cooperative Research Program of Institute for Protein Research, Osaka University

\section{REFERENCES}

[1] E. C. Horning, V. L. Stromberg, H. A. Lloyd, J. Am. Chem. Soc. 1952, 74, 5153-5155.

[2] M. Boero, T. Ikeshoji, C. C. Liew, K. Terakura, M. Parrinello, J. Am. Chem. Soc. 2004, 126, $6280-6286$

[3] S. Kinoshita, S. Kageyama, K. Iba, Y. Yamada, and H. Okada, Agric. Biol. Chem., 1975, 39, $1219-1223$.

[4] H. Okada, S. Negoro, H. Kimura, S. Nakamura, Nature, 1983, 306, 203-206.

[5] K. Kato, K. Ohtsuki, Y. Koda, T. Maekawa, T. Yomo, S. Negoro, I. Urabe, Microbiol., 1995, $141,2585-2590$.

[6] S. Negoro, Appl. Microbiol. Biotechnol., 2000, 54, 461-466.

[7] K. Nagai, Iida, K. Shimizu, R. Kinugasa, M. Izumi, D-I, Kato, M. Takeo, K. Mochiji, and S. Negoro, Appl. Microbiol. Biotechnol., 2014, DOI:10.1007/s00253-014-5885-2. -in press 
[8] S. Negoro, N. Shibata, Y. Tanaka, K. Yasuhira, H. Shibata, H. Hashimoto, Y. Lee, S. Oshima, R. Santa, S. Oshima, K. Mochiji, Y. Goto, T. Ikegami, K. Nagai, D. Kato, M. Takeo, and Y. Higuchi, J. Biol. Chem., 2012, 287, 5079-5090.

[9] S. Negoro, T. Ohki, N. Shibata, N. Mizuno, Y. Wakitani, J. Tsurukame, K. Matsumoto, I. Kawamoto, M. Takeo, and Y. Higuchi, J. Biol. Chem., 2005, 280, 39644-39652.

[10] K. Kato, K. Fujiyama, H.S. Hatanaka, I.D. Priyambada, S. Negoro, I. Urabe, and H. Okada, Eur. J. Biochem. 1991, 200, 165-169.

[11] S. Negoro, T. Ohki, N. Shibata, K. Sasa, H. Hayashi, H. Nakano, K. Yasuhira, D. Kato, M.Takeo, and Y. Higuchi, J. Mol. Biol., 2007, 370, 142-156.

[12] T. Ohki, N. Shibata, Y. Higuchi, Y. Kawashima, M. Takeo, D. Kato, and S. Negoro, Protein Sci. 2009, 18, 1662-1673.

[13] Y. Kawashima, T. Ohki, N. Shibata, Y. Higuchi, Y. Wakitani, Y. Matsuura, Y. Nakata, M. Takeo, D. Kato, and S. Negoro, FEBS J., 2009, 276, 2547-2556.

[14] P. H. Crowley, J. Theo. Biol., 1975, 50, 461-475.

[15] K. A. Johnson, and R. S. Goody, Biochem., 2011, 50, 8264-8269.

[16] K. Kamiya, T. Baba, M. Boero, T. Matsui, S. Negoro, and Y. Shigeta, J. Phys. Chem. Lett., 2014, $5,1210$.

[17] H. M. Senn, and W. Thiel. Angew. Chem. Int. Ed., 2009, 48, 1198-1229.

[18] G. Dong, S. Shaik, and W. Lai, Chem. Sci., 2013, 4, 3624-3635

[19] R. Harada, A. Kitao, J. Chem. Phys. 2013, 139, 035103.

[20] T. Baba, R. Harada, M. Nakano, and Y. Shigeta, J. Comput. Chem., 2014, 35, 1240-1247.

[21] S. Tanaka, Y. Mochizuki, Y. Komeiji, Y. Okiyama, and K. Fukuzawa, Phys. Chem. Chem. Phys., 2014, 16, 10310-10344.

[22] K. Fukuzawa, C. Watanabe, I. Kurisaki, N. Taguchi, Y. Mochizuki, T. Nakano, S. Tanaka, and Y. Komeiji, Comput. Theor. Chem., 2014, 1034, 7-16.

[23] C. Watanabe, K. Fukuzawa, S. Tanaka, and S. Aida-Hyugaji, J. Phys. Chem. B, 2014, 118, 4993-5008.

[24] W. L. Jorgensen, J. Chandrasekhar, and J. D. Madura, J. Comput. Phys., 1983, 79, 926. 
[25] D.A. Case, V. Babin, J.T. Berryman, R.M. Betz, Q. Cai, D.S. Cerutti, T.E. Cheatham, III, T.A. Darden, R.E. Duke, H. Gohlke, A.W. Goetz, S. Gusarov, N. Homeyer, P. Janowski, J. Kaus, I. Kolossváry, A. Kovalenko, T.S. Lee, S. LeGrand, T. Luchko, R. Luo, B. Madej, K.M. Merz, F. Paesani, D.R. Roe, A. Roitberg, C. Sagui, R. Salomon-Ferrer, G. Seabra, C.L. Simmerling, W. Smith, J. Swails, R.C. Walker, J. Wang, R.M. Wolf, X. Wu and P.A. Kollman, 2014, AMBER 14, University of California, San Francisco.

[26] R. Salomon-Ferrer, A.W. Goetz, D. Poole; S. Le Grand, and R.C. Walker, J. Chem. Theory Comput., 2013, 9, 3878-3888.

[27] T. Baba, K. Kamiya, T. Matsui, N. Shibata, Y. Higuchi, T. Kobayashi, S. Negoro, and Y. Shigeta, Chem. Phys. Lett., 2011, 507, 157-161.

[28] J.-P. Ryckaert, G. Ciccotti, and H. J. C. Berendsen, J. Comput. Phys., 1977, 23, 327-341

[29] T. Darden, D. York, and L. Pedersen, J. Chem. Phys. 1993, 98, 10089.

[30] H. Li, A. D. Robertson, and J. H. Jensen, Proteins, 2005, 61, 704-721.

[31] D. C. Bas, D. M. Rogers, and J. H. Jensen, Proteins, 2008, 73,765-783.

[32] M. H M. Olsson, C. R. Søndergard, M. Rostkowski, and J. H. Jensen, J. Chem. Theory Comput., 2011, 7, 525-537.

[33] C. R. Søndergaard, M. H. Olsson, M. Rostkowski, and J. H. Jensen, J. Chem. Theory Comput., 2011, 7, 2284-2295.

[34] A. Laio, J. VandeVondele, and U. Roethlisberger, J. Chem. Phys. 2002, 116, 6941-6947.

[35] A. Laio, J. VandeVondele, and U. Roethlisberger, J. Phys. Chem. B 2002, 106, 7300-7307.

[36] A. Laio, and M. Parrinello, Proc. Natl. Acad. Sci. U. S. A., 2002, 99, 12562-12566.

[37] M. Iannuzzi, A. Laio, and M. Parrinello, Phys. Rev. Lett., 2003, 90, 238302.

[38] A. Barducci, M. Bonomi, and M. Parrinello, Comput. Mol. Sci., 2011, 826-843.

[39] M. Bonomi, D. Branduardi, G. Bussi, C. Camilloni, D. Provasi, P. Raiteri, D. Donadio, F. Marinelli, F. Pietrucci, R. A. Broglia, and M. Parrinello, Comput. Phys. Commun., 2009, 180, 1961-1972.

[40] F. Pietrucci, F. Marinelli, P. Carloni and A. Laio J. Am. Chem. Soc., 2009, 131, 11811-11818.

[41] R. Car, and M. Parrinello, Phys. Rev. Lett., 1985, 55, 2471-2474.

[42] P. Hohenberg, and W. Kohn, Phys. Rev., 1964, 136, B864-B871. 
[43] W. Kohn, and L.J. Sham, Phys. Rev., 1965, 140, A1133-A1138

[44] F. L. Gervasio, M. Boero, and M. Parrinello, Angew. Chem. Int. Ed., 2006, 45, 5606-5609.

[45] M. Boero, T. Ikeda, E. Ito, and K. Terakura, J. Am. Chem. Soc., 2006, 128, 16798-16807.

[46] K. Kamiya, M. Boero, M. Tateno, K. Shiraishi, and A. Oshiyama, J. Am. Chem. Soc., 2007, 129, 9663-9673.

[47] M. Boero, J. Phys. Chem. B, 2011, 115, 12276.

[48] X. Biarnés, A. Ardevol, J. Iglesias-Fernández, A. Planas, and C. Rovira, J. Am. Chem. Soc., 2011, 133, 20301-20309

[49] F. Hofbauer, and I. Frank, Chem. Eur. J., 2012, 18, 16332-16338.

[50] Rovira, C. Comput. Mol. Sci., 2013, 393-407.

[51] M. Boero, Lecture Notes in Physics, 2010, 795, 81-98.

[52] CPMD, http://www.cpmd.org/, Copyright IBM Corp 1990-2012, Copyright MPI für Festkörperforschung 1997-2001.

[53] F.A. Hamprecht, A.J. Cohen, D.J. Tozer, and N.C. Handy, J. Chem. Phys., 1998, 109, $6264-6271$.

[54] N. Troullier, and J.L. Martins, Phys. Rev. B, 1991, 43, 1993-2006.

[55] A. Laio and F. L. Gervasio, Rep. Prog. Phys. 2008, 71, 126601.

[56] W. Humphrey, A. Dalke, and K. Schulten, J. Mol. Graphics, 1996, 14, 33.

[57] A. Grossfield, WHAM: The Weighted Histogram Analysis Method, Version 2.07. Available at: http://membrane.urmc.rochester.edu/content/wham. Accessed September 22, 2014.

[58] D. Hankins, J. W. Moskowiz, F. H. Strillinger, J. Chem. Phys., 1970, 53 4544-4554.

[59] T. Nakano, T. Kaminuma, T. Sato, K. Fukuzawa, Y. Akiyama, M. Uebayasi, K. Kitaura, Chem. Phys. Lett., 2002, 351, 475-480.

[60] Y. Mochizuki, K. Yamashita, K. Fukuzawa, K. Takamatsu, H. Watanabe, N. Taguchi, Y. Okiyama, M Tsuboi, T Nakano, S Tanaka, Chem. Phys. Lett., 2010, 493, 346-352.

[61] H. Ando, Y. Shigeta, T. Baba, C. Watanabe, Y. Okiyama, Y. Mochizuki, and M. Nakano, Mol. Phys., 2014, DOI:10.1080/00268976.2014.941311. - in press

[62] Y. Komeiji, T. Ishida, D. G. Fedorov, and K. Kitaura, J. Comput. Chem., 2007, 28,1750-1762. 
[63] BioStation Viewer, A. Kato, K. Fukuzawa, T. Nakano, Available at: http://www.fsis.iis.u-tokyo.ac.jp/en/result/software/. September 22, 2014.

[64] P.-O. Syrén, FEBS Journal 2013, 280, 3069-3083.

[65] K. S. Werpetinsk, and M. Cook. Phys. Rev., 1995, 52, R3397.

[66] H. Lizbeth. Chem. Rev., 2002, 102, 4501-4524.

[67] V. Daggett, S. Schroeder, and P. Kollman, J. Am. Chem. Soc., 1991, 113, 8926-8935.

[68] T. Ishida, and S. Kato. J. Am. Chem. Soc., 2003, 125, 12035-12048.

[69] L. Holm and P. Rosenström. Nucleic Acids Res., 2010, 38, W545-W549.

[70] S. S. Cha, Y. J. An, C. S. Jeong, M. K. Kim, J. H. Jeon, C. M. Lee, H. S. Lee, S. G. Kang, and J. H. Lee, Proteins: Struct. Funct. Bioinfo., 2013, 81, 2045-2051.

[71] E. Roberts, J. Eargle, D. Wright, and Z. Luthey-Schulten,s

[72] R. B. Russell and G. J. Barton, PROTEINS: Struct. Funct. Genet., 1992, 14, 309-323.

[73] D. J. Tantillo, C. Jiangang, and K. N. Houk, Curr. Opin. Chem. Biol., 1998, 2, 743-750.

[74] D. Röthlisberger, O. Khersonsky, A. M. Wollacott, L. Jiang, J. DeChancie, J. Betker, J. L. Gallaher, E. A. Althoff, A. Zanghellini, O. Dym, S. Albeck K. N. Houk, D. S. Tawfik, and D. Baker, Nature, 2008, 453, 190-195.

[75] H. K. Privett, G. Kiss, T. M. Lee, R. Blomberg, R. A. Chica, L. M. Thomas, D. Hilvert, K. N. Houk, and S. L. Mayo, Proc. Natl. Acad. Sci. USA, 2012, 109, 3790-3795.

[76] J. H. Mills, S. D. Khare, J. M. Bolduc, F. Forouhar, V. K. Mulligan, S. Lew, J. Seetharaman, L. Tong, B. L. Stoddard and D. Baker, J. Am. Chem. Soc., 2013, 135, 13393-13399.

[77] S. Bjelic, Y. Kipnis, L. Wang, Z. Pianowski, S. Vorobiev, M. Su, J. Seetharaman, R. Xiao, G. Kornhaber, J. F. Hunt, L. Tong, D. Hilvert, and D. Baker, J. Mol. Biol., 2014, 426, 256-271.

[78] A. Radzicka, and R. Wolfenden, Science, 1995, 267, 90-93.

[79] W. S. Mak and J. B. Siegel Curr. Opin. Struct. Biol., 2014, 27 87-94.

[80] L. S. Mizoue, and W. J. Chazin. Curr. Opin. Struct. Biol., 2002, 12, 459-463.

[81] H. I. Ingólfsson, C. A. Lopez, J. J. Uusitalo, D. H. de Jong, S. M. Gopal, X. Periole, and S. J. Marrink, WIREs: Comput. Mol. Sci., 2014, 4, 225-248.

[82] R. Harada, Y. Takano, and Y. Shigeta. J. Chem. Phys, 2014, 140, 125103.

[83] B. G. Johnson, P. M. W. Gill, and J. A. Pople, J. Chem. Phys. 1993, 98, 5612-5626. 


\section{Figure Captions}

Figure 1. Structure and main amino acid residues of NylB. (a) Induced-fit process: Superposition of the NylB structures in the open form (black) and in the closed form (yellow). A large movement of the loop-segment N166-V177 toward the substrate occurs, indicated by the red arrow enclosed in the dotted red circle. (b) The QM region, located inside the red circle in (a), used in our hybrid QM/MM simulations. QM atoms are shown as balls and sticks, whose colors code, here and in the all the following figures, are gray for $\mathrm{C}$, red for $\mathrm{O}$, blue for $\mathrm{N}$ and white for $\mathrm{H}$, and the yellow ribbon shows the secondary structure. (c) The reaction coordinate (red arrow) and other fundamental distances (green, blue and magenta) used to drive and monitor the acylation reaction. Details are given in the text and in Fig. 2.

Figure 2. Evolution of the reaction coordinate $r_{1}$ and the most relevant distances during the metadynamics simulation for the Y170F mutant: $r_{1}=\mathrm{N} 3\left(\right.$ Ald393)-C1(Ald393) (red), $r_{2}=$ OG(Ser112)-C1(Ald393) (green), $\quad r_{3}=\mathrm{HH}\left(\right.$ Tyr215)- N3(Ald393) (blue), and $r_{4}=$ HG(Ser112)-NZ(Lys115) (magenta). Panel (a) shows whole simulation, while panels (b), (c), and (d) are the magnifications of the three crucial events inside the dashed circles in (a).

Figure 3. Local structures and related differences between Y170F and WT relevant to the acylation process. Panels (a), (b), and (c) correspond, respectively, to the events (b), (c), and (d) in Fig. 2. Panel (d) shows the WT for comparison. A schematic picture of the difference between Y170F and WT is shown in (e).

Figure 4. Free energy landscapes for the induced-fit process in the Y170F mutant(a) and the WT (b), according to the results of Ref. 20.

Figure 5. (a) Schematic view of the interactions between the most relevant amino acid residues and the substrate. For clarity, the ALD is divided into three fragments, the C-terminal, the peptide site, and the N-terminal, labeled as $\operatorname{ALD}(1)$ (blue), $\operatorname{ALD}(2)$ (pink), and $\operatorname{ALD}(3)$ (green), respectively. These fragments are the ones used in the FMO analysis shown in panel (b). 
Figure 6. Result of the structural homology search (a) and details of the surrounding substrate (b) Using the DALI program and selecting only structures preserving the local environment around Tyr170 in WT (pdb id 2ZMA), EstU1 (pdb id 4IVK) was discovered. For the structural aliment, we adopted the STAMP algorithm. The corresponding analogous main regions between WT and EstU1 are shown in the two adjacent panels in (a). The semi-transparent elliptical region on the top left of each structure indicates the two segments 179-189 of 4IVK, and 175-185 of 2ZMA, respectively. Each substrate is shown as yellow sticks.

Table 1. Maximum free energy differences in the acylation step for the WT and the Y170F mutant. We recall that typical error bars of $1-2 \mathrm{kcal} / \mathrm{mol}$ from the underlying level of DFT adopted affect these estimations

\begin{tabular}{c|c}
\hline System & Maximum free energy difference $\Delta G^{\mathrm{a}}$ \\
\hline WT & 31 \\
Y170F mutant & 42 \\
\hline \multicolumn{2}{c}{} \\
\multicolumn{2}{c}{${ }^{\mathrm{a}}$ unit of $\mathrm{kcal} / \mathrm{mol}$}
\end{tabular}

Table 2. The computed IFIE values between the $170^{\text {th }}$ and the $181^{\text {st }}$ amino acid residues and each fragment in Ald.

\begin{tabular}{cccccc}
\hline \multirow{2}{*}{ System } & Residue & \multicolumn{5}{c}{ IFIE $^{\mathrm{a}}$} \\
\cline { 3 - 6 } & & ALD(1) & ALD(2) & ALD(3) & Total \\
\hline \multirow{2}{*}{ WT $^{\mathrm{b}}$} & Tyr170 & -2.07 & -6.01 & 0.73 & -7.35 \\
& Asp181 & 18.31 & -2.63 & -115.27 & -99.59 \\
& Phe170 & -4.69 & 0.33 & -1.44 & -5.80 \\
& Asp181 & 20.19 & -3.05 & -118.21 & -101.07 \\
& Tyr170 & -4.87 & -4.79 & -4.34 & -14.00 \\
& Glyb-24 & -0.52 & 0.13 & 1.22 & 0.83 \\
\hline
\end{tabular}

a unit of $\mathrm{kcal} / \mathrm{mol},{ }^{\mathrm{b}}$ From Ref. 59 

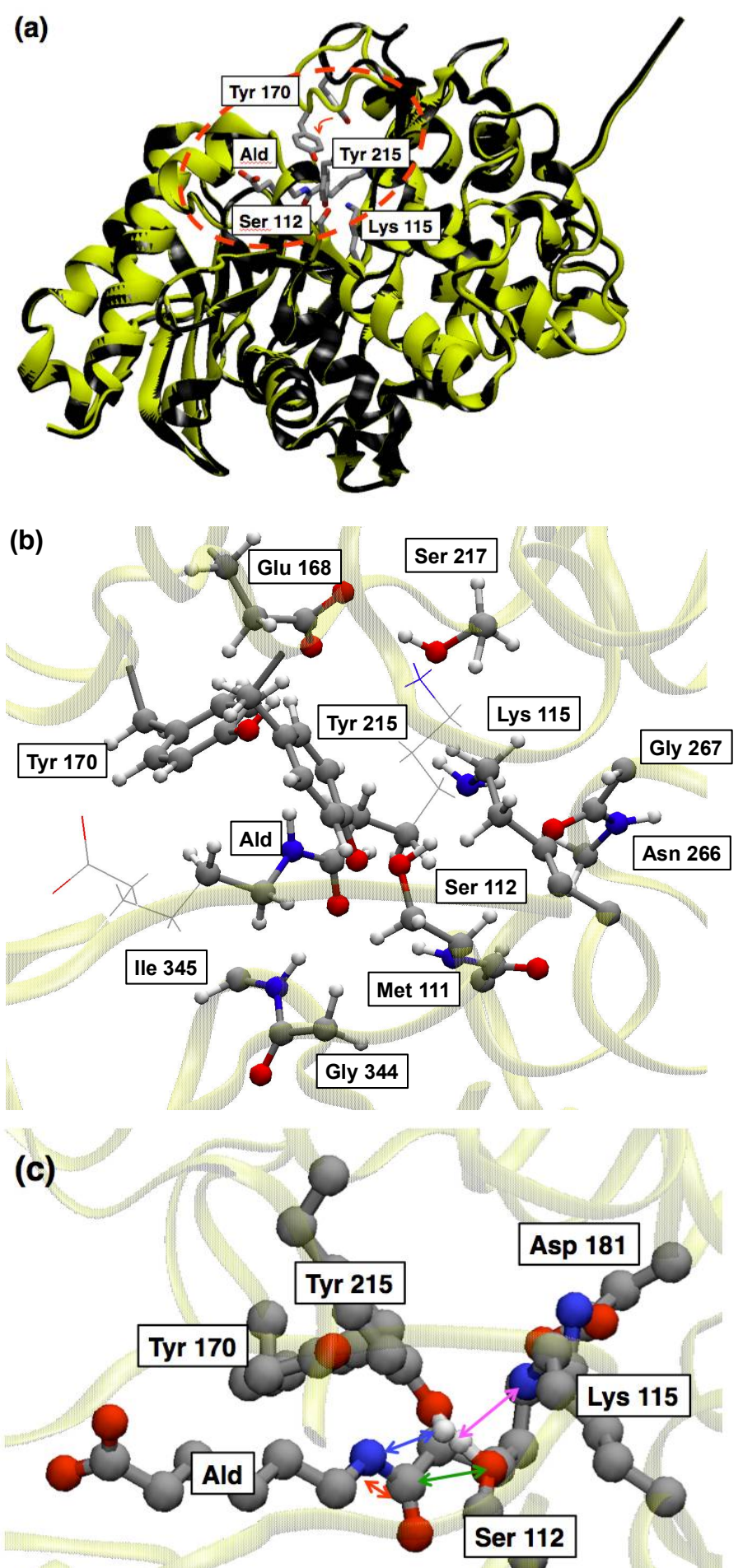

Figure 1 T. Baba et al. 
(a)

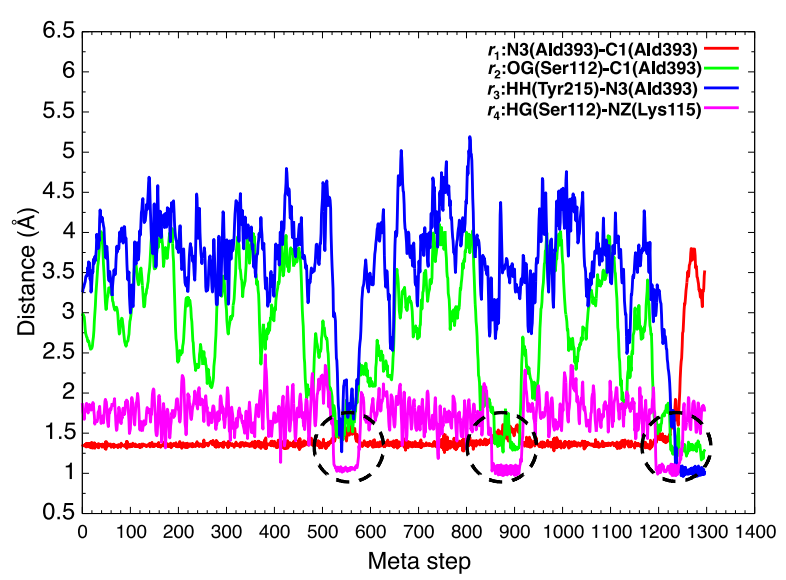

(c)

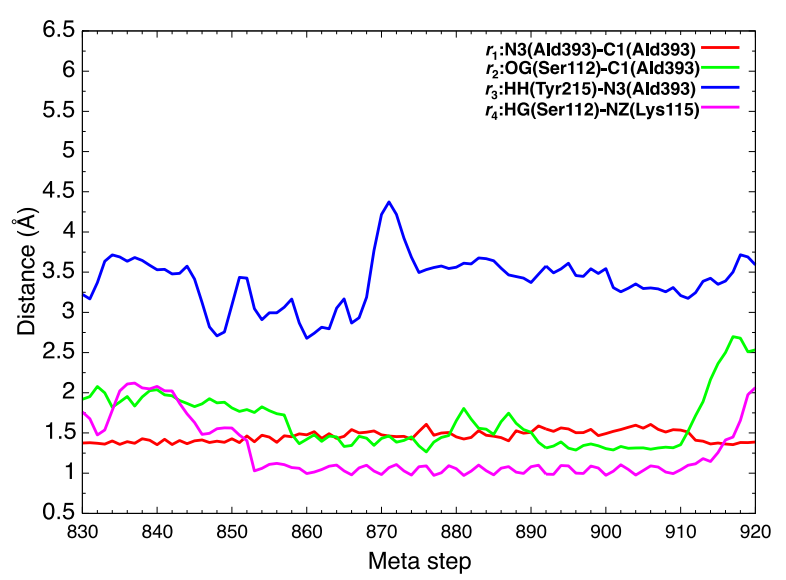

(b)

(d)
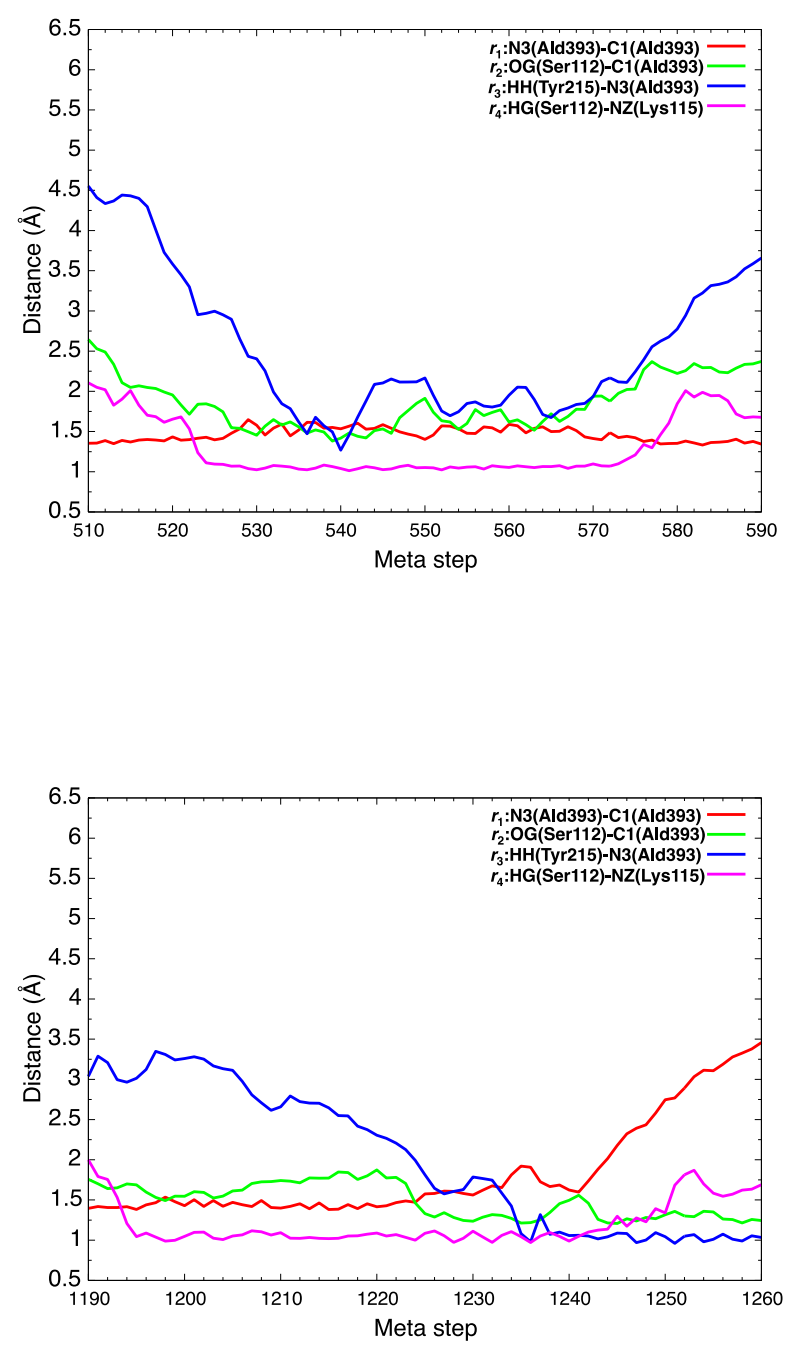

Figure 2 T. Baba et al. 

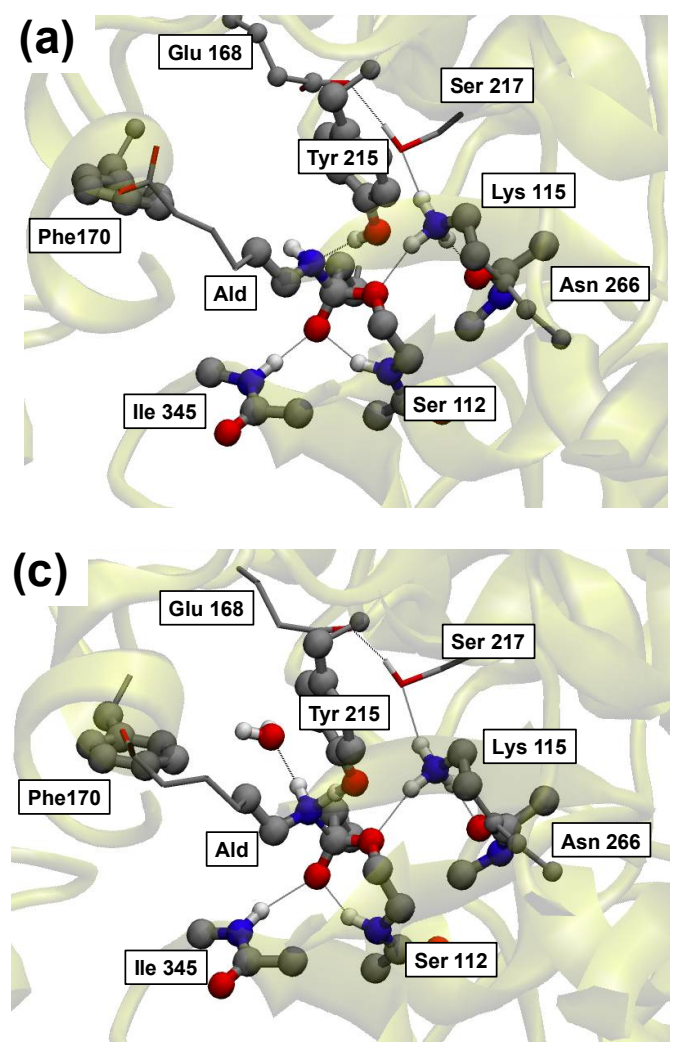

(e)

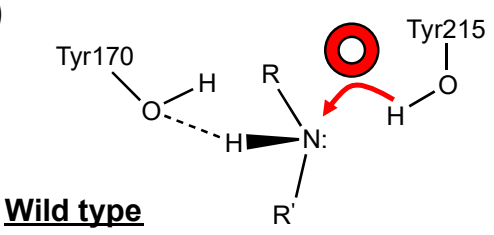

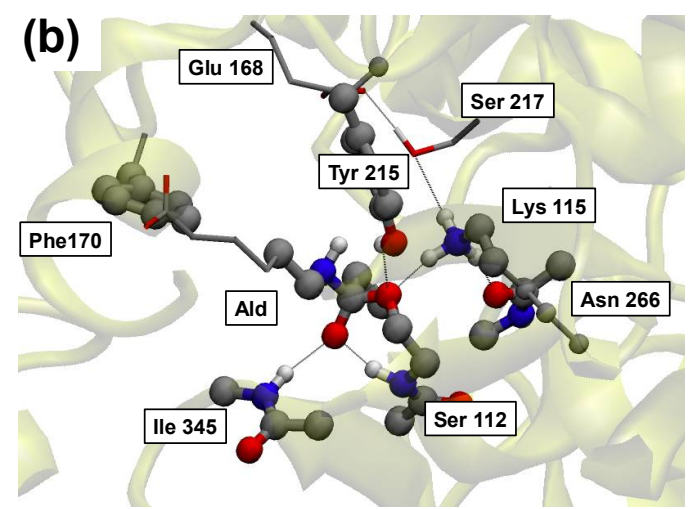
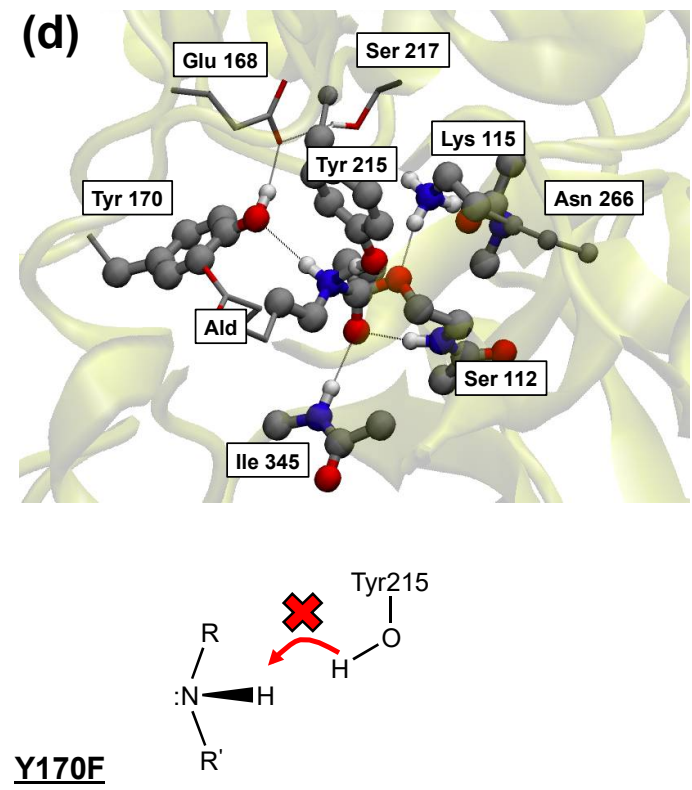

Figure 3 T. Baba et al. 
(a)

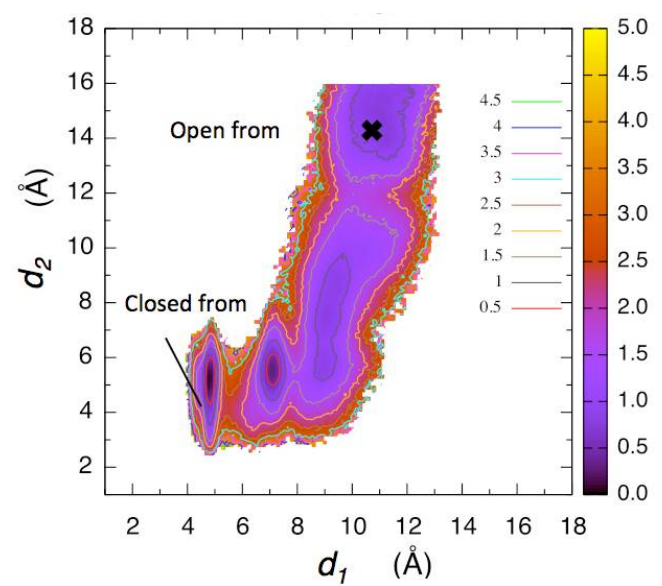

(b)

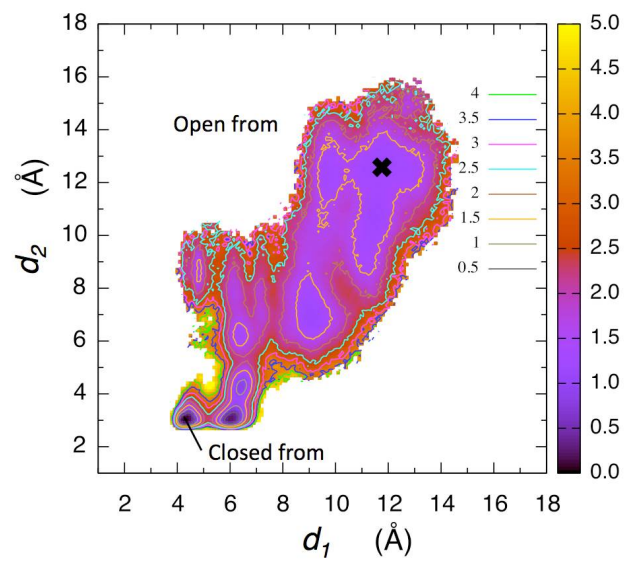

Figure 4 T. Baba et al. 
(a)

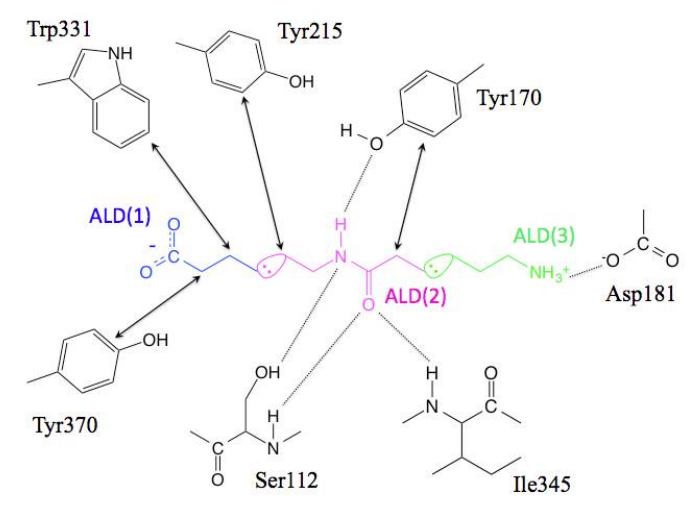

(b)
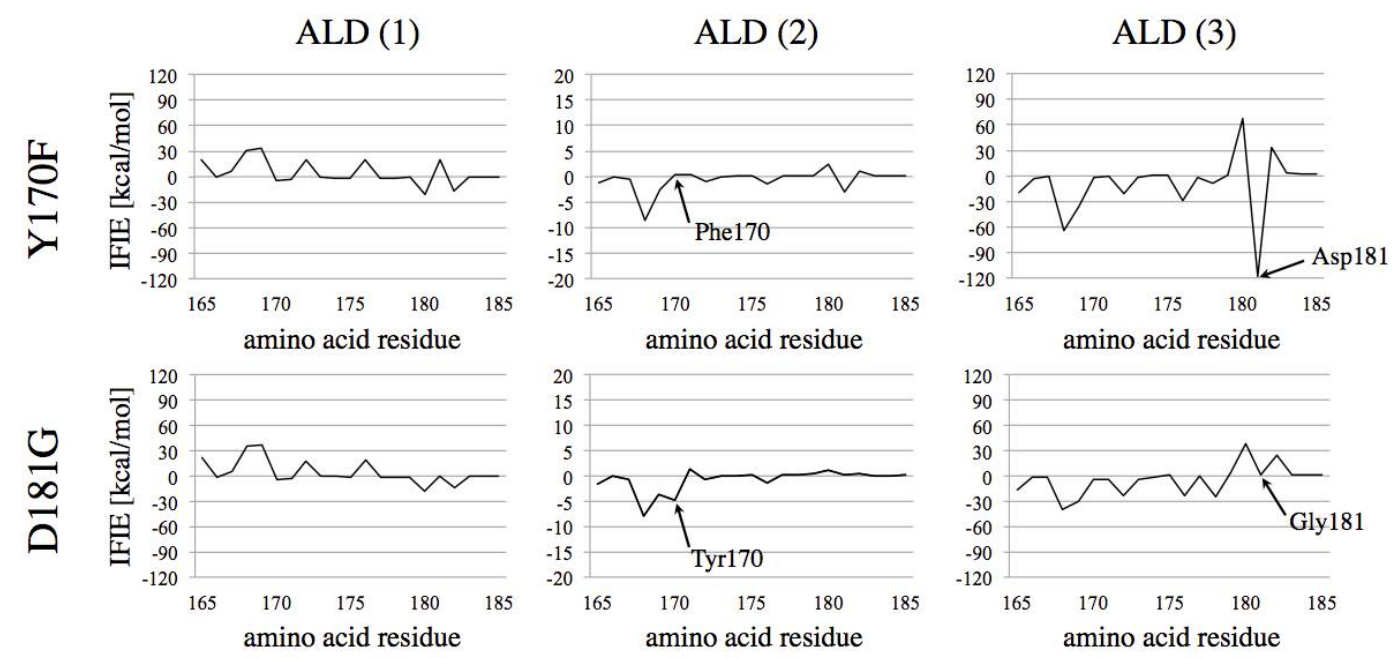

Figure 5 T. Baba et al. 
(a)
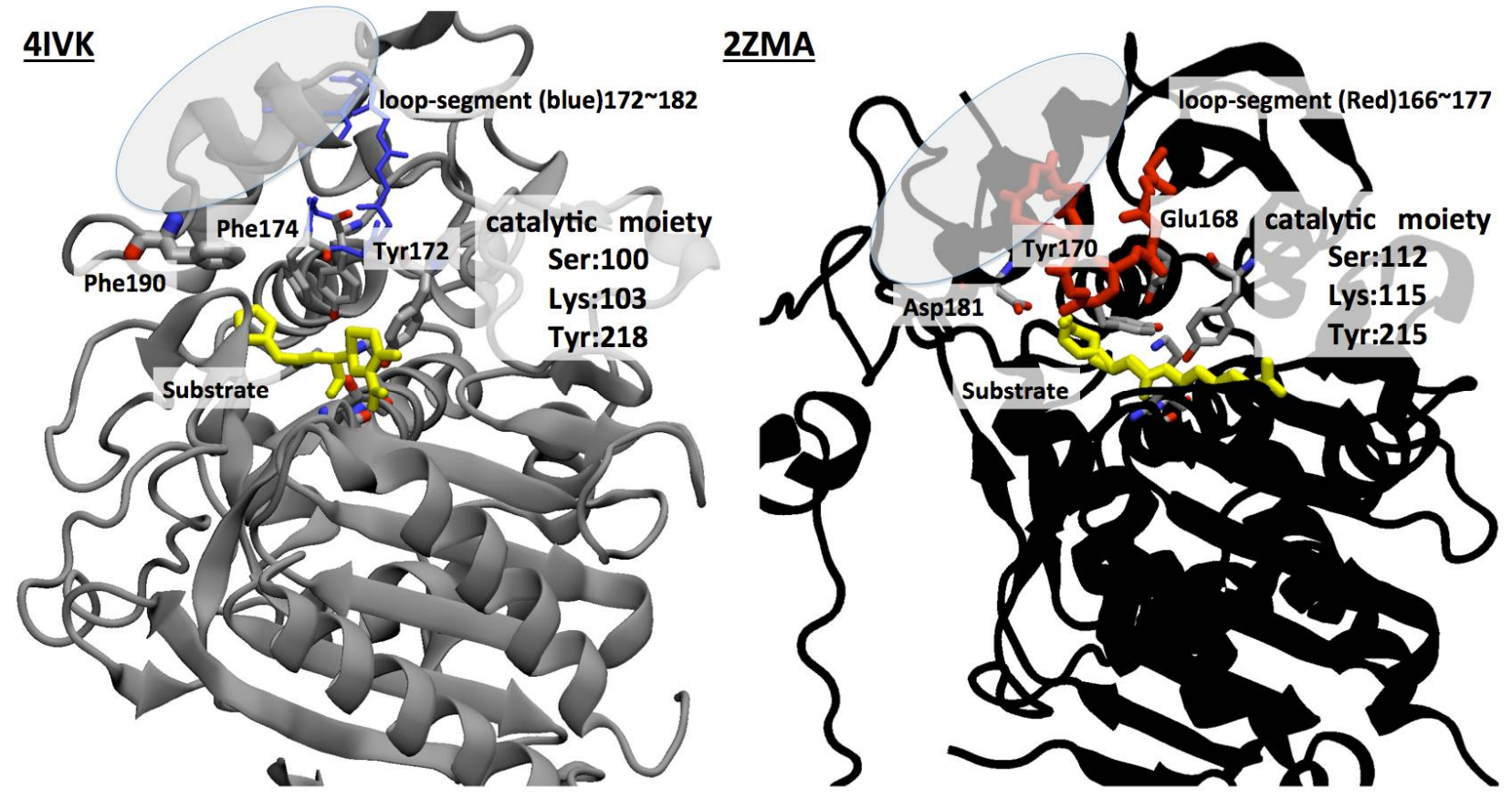

(b)

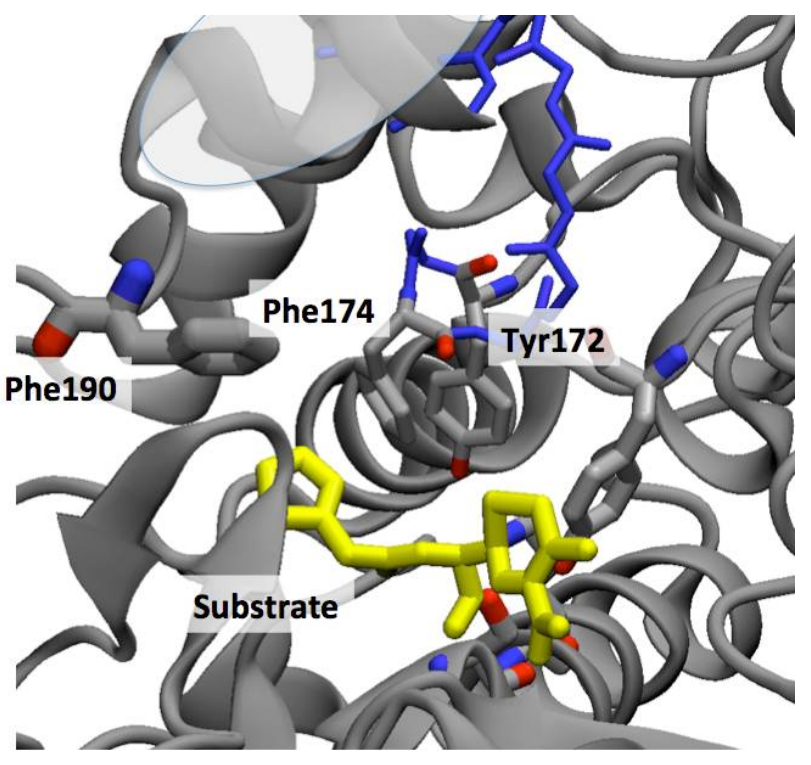

Figure 6 T. Baba et al. 


\section{Table of contents}

To unravel what provides the ability to degrade non-biological amide bond with Nylon oligomer hydrolase, we investigate the process from induced-fit to acylation by a combination of different theoretical methods.

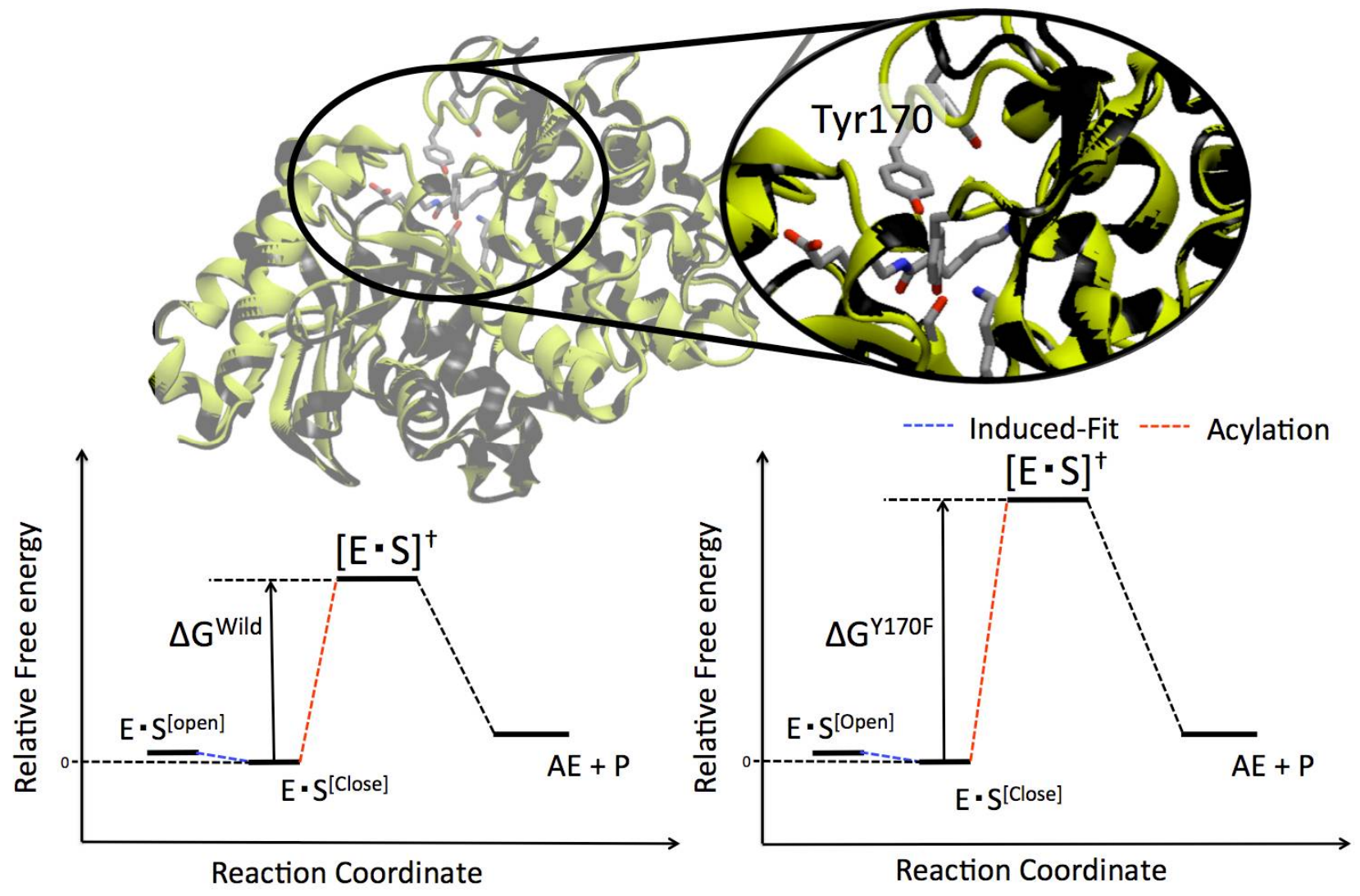

